Artículo

\title{
Presencia de Hymenolepis nana y diminuta en roedores de la ciudadela las Piñas, Milagro-Ecuador y su riesgo en salud pública
}

Roberto Darwin Coello-Peralta ${ }^{\text {a }}$

Galo Ernesto Martínez-Cepeda ${ }^{\text {a* }}$

Douglas Pinela-Castro $^{\text {a }}$

Enrique Omar Reyes-Echeverria ${ }^{a}$

Enrique Xavier Rodríguez-Burnham ${ }^{\text {a }}$

Maria de Lourdes Salazar Mazamba ${ }^{\text {a }}$

Betty Pazmiño-Gómez ${ }^{\text {b }}$

Antonella Ramírez-Tigrero ${ }^{\text {a }}$

Manuel Bernstein ${ }^{a}$

Pedro Cedeño-Reyes ${ }^{\text {a }}$

${ }^{a}$ Universidad de Guayaquil. Facultad de Medicina Veterinaria y Zootecnia. Km 27 1⁄2 vía a Daule, Guayaquil, Ecuador.

${ }^{\mathrm{b}}$ Universidad Estatal de Milagro. Facultad Ciencias de la Salud, Milagro, Ecuador.

*Autor de correspondencia: galomartinez88@gmail.com

\section{Resumen:}

La Hymenolepidiosis es una zoonosis de prevalencia mundial, sobre todo en niños, es causada por cestodos de roedores denominados Hymenolepis (H) nana e Hymenolepis diminuta, es muy frecuente en países en vías de desarrollo, con climas cálidos, templados y secos. El ciclo biológico de la $H$. nana no requiere de hospederos intermediarios, y su transmisión habitual es fecal-oral (por ingesta de huevos infectivos); y la infección de $H$. diminuta se da a través de la ingestión de artrópodos tenebriónidos con la forma larvaria cisticercoides. El objetivo de este estudio fue determinar la presencia de $H$. nana e $H$. 
diminuta en la ciudadela Las Piñas, de la ciudad de Milagro (Ecuador) y dar a conocer a través de charlas informativas el riesgo en salud pública; para esta investigación se capturaron roedores con ayuda de trampas Tomahawk y Sherman en complementariedad con cebos no tóxicos (carne, mortadela, pescado, pan). Mediante un estudio aplicado, con enfoque cualitativo, de tipo descriptivo-prospectivo-transversal, realizado entre el 1 de febrero al 30 de julio del 2018, se analizaron las muestras fecales por métodos directos y de flotación-centrifugación con solución salina sobresaturada. De 87 roedores capturados y procesados, 20 casos (22.99 \%) se determinó para Hymenolepis nana y 10 casos $(11.49 \%)$ para $H$. diminuta. Constituyéndose en el primer reporte de Hymenolepis nana y diminuta en roedores en el país. Se concluye que es evidente la presencia de estos parásitos en el sitio de estudio, lo que podría convertirse en un serio problema de salud pública, por el riesgo de transmitirse a los habitantes del sector.

Palabras clave: Trampeo, Métodos coproparasitarios, Parasitismo en roedores, Salud pública.

Recibido: 05/12/2018

Aceptado: 03/09/2019

\section{Introducción}

Los agentes infecciosos transmitidos de animales a humanos representan la mayoría de los brotes de nuevos patógenos en todo el mundo ${ }^{(1)}$, con más de mil millones de casos de enfermedades humanas atribuibles a enfermedades zoonóticas cada año, por lo cual, la identificación de reservorios silvestres de patógenos zoonóticos es una prioridad en Salud Pública ${ }^{(2)}$.

En el caso de Rattus norvegicus (rata marrón) y Rattus rattus (rata negra) son reservorios conocidos de bacterias, virus y parásitos zoonóticos de importancia Veterinaria ${ }^{(3,4)}$. Las helmintosis afectan al $20 \%$ de la población latinoamericana y se consideran enfermedades desatendidas, que infectan aproximadamente a 3,800 millones de personas en todo el mundo ${ }^{(5)}$. Estas infecciones son frecuentes en poblaciones rurales y en zonas superpobladas, sobre todo en países en clima tropical y subtropical, asociadas con la pobreza y marginación y no reciben la debida atención nacional e internacional ${ }^{(6,7)}$.

Entre los factores sociales determinantes de dichas enfermedades incluyen deficientes condiciones de vivienda (sin techos, paredes o pisos adecuados), la falta de acceso a agua potable y segura, saneamiento básico e higiene, los bajos ingresos, la educación deficiente y las barreras de acceso a los servicios de salud en general y, en especial, a la atención primaria en salud ${ }^{(7,8)}$.

La Himenolepiasis es una parasitosis de prevalencia mundial causada por cestodos del género Hymenolepis y Rodentolepis (Hymenolepipidae: Cyclophyllidea). Los ciclos de 
vida de estos parásitos implican humanos, ratas y ratones como huéspedes definitivos, y artrópodos (Tenebriónidos) como huéspedes intermedios. La presencia de tenebriónidos en las viviendas y condiciones de insalubridad generadas por la falta de servicios básicos, factores ambientales y socio-económicos, los cuales componen un factor predisponente para el desarrollo de la enfermedad en áreas cálidas y húmedas; causando mayores afectaciones a la población infantil ${ }^{(9,10)}$.

Las ratas y ratones actúan como hospedadores definitivos de Hymenolepis y su ocurrencia de esta parasitosis en humanos es habitualmente transmitido por vía fecal-oral por ingesta de huevo (H. nana); sin embargo, ocasionalmente también puede transmitirse por ingestión de un artrópodo para completar su ciclo de vida $(H \text {. diminuta })^{(11,12)}$.

Se estima que 20 millones de personas en el mundo están parasitadas con estos dos parásitos $^{(13)}$. Sin embargo, ambas parasitosis comparten algunas características epidemiológicas, tales como prevalencia en niños de áreas marginadas con malos hábitos de higiene, condiciones sanitarias y hacinamiento ${ }^{(12)}$.

Ecuador es un país con gran variedad de climas con cuatro regiones delimitadas: costa, sierra, oriente y región insular. La ciudadela "Las Piñas", se encuentra localizada en la costa ecuatoriana, se caracteriza por tener un clima tropical además de altos porcentajes de humedad debido a los ríos y planicies que posee. La ciudadela mencionada posee un sector urbano marginal con 2,500 viviendas, en donde se encuentra: casas desocupadas, sembríos de caña, cacao, banano y pastizales; además, se encuentran zanjas, pozos sépticos; por otra parte, no posee alcantarillado, pocos hogares poseen agua potable, por lo consiguiente, reúne las condiciones necesarias para contar con la presencia de roedores que representan un riesgo sanitario ${ }^{(14)}$. Además, en el país no se ha reportado la presencia de Hymenolepis nana y diminuta en roedores, por lo cual, es necesario dar a conocer casos de estas parasitosis, ya que son potenciales fuentes de parásitos, que podrían conllevar a problemas de salud pública por su transmisión a los humanos. Por otra parte, el objeto de este estudio es informar a las autoridades de salud pública y profesionales de la salud sobre casos de Hymenolepidiasis en roedores, además de informar a la población y guiarlos en los esfuerzos de prevención.

\section{Material y métodos}

\section{Área y tiempo de estudio}

El estudio se realizó en la ciudadela urbano-marginal llamada "Las Piñas", ubicada en la parte norte del cantón Milagro, provincia del Guayas, en la costa ecuatoriana, sus coordenadas geográficas son $2^{\circ} 7^{\prime} 0^{\prime \prime} \mathrm{S}$ y $79^{\circ} 36^{\prime} 0^{\prime \prime} \mathrm{O}$. La comunidad "Las Piñas" posee 12,000 habitantes, cuenta con un clima tropical-húmedo, con marcada diferencia entre el invierno y verano, con temperaturas que fluctúan entre los 22 a $36{ }^{\circ} \mathrm{C}^{(14,15)}$. 


\section{Encuesta}

Se visitó cada uno de los hogares seleccionados, se explicó sobre el estudio y el riesgo que tienen las parasitosis en su entorno, luego de su consentimiento, y asentimiento firmado (para menores), se aplicó una encuesta dirigida, y posterior se entregó un frasco estéril por cada persona.

La encuesta presentó las siguientes preguntas: ¿Conoce sobre la hymenolepidiasis? ¿Cuál es el origen del agua de consumo?, ¿Cuál es el manejo de excretas dentro del hogar?, ¿Existe la presencia de roedores intra y peridomiciliarios?, ¿Tiene depósitos de alimentos farináceos (arroz, maíz, cebada, etc)?, ¿Hay presencia de Tenebriónidos (escarabajos, pulgas, gorgojos, etc) en los depósitos de alimentos farináceos? y ¿ Tiene alcantarillado?.

\section{Muestreo}

Previamente a realizar el estudio se solicitó el respectivo permiso a las autoridades de la Facultad de Medicina Veterinaria y Zootecnia (FMVZ) de la Universidad de Guayaquil para que acceda a dar el permiso respectivo para realizar el estudio en el laboratorio de Microbiología de la FMVZ; también, contó con el permiso y colaboración del Comité Pro mejoras "Nuevos Horizontes de Las Piñas". Por otro lado, la metodología de la investigación fue analizada y aprobada por la Coordinación de Investigación de la FMVZ.

Se realizó un estudio descriptivo, transversal y prospectivo, entre el 1 de febrero al 31 de julio del 2018; se investigaron 70 viviendas con una población total de 320 personas, entre invierno y verano, con temperatura aproximada de 27 a $32{ }^{\circ} \mathrm{C}$.

Durante la investigación se muestrearon 87 roedores, provenientes de tres áreas seleccionadas 1) roedores en casas habitadas, solares baldíos, zanjas, y basureros, 2) zanjas y basureros, y 3) Unidad Policial Comunitaria (UPC), zanjas, basureros y solares baldíos. Es importante mencionar que todas las áreas investigadas registraron concentraciones de basura. Por otra parte, se realizó la encuesta a 90 personas del sector, dividido en 30 por cada sector ya mencionado.

\section{Captura de roedores}

Se realizaron capturas de roedores con ayuda de trampas, Tomahawk y Sherman, y de cebos no tóxicos (carne, mortadela, pescado, pan) tomando medidas de bioseguridad recomendadas. Estas trampas se colocaron de forma estratégica en "Las Piñas" la cual es una ciudadela urbanomarginal, la cual posee lugares con riesgo como: botaderos de basuras, zanjas, pastizales, y en las casas de ciertos colaboradores de la comunidad que aseguran tener roedores ${ }^{(16)}$.

Aquellos roedores capturados vivos fueron sacrificados con cloroformo puro empapado en algodón para la eutanasia; cuyo método es el sugerido por el CICUAL de la Facultad 
de Medicina Veterinaria y Zootecnia de la Universidad de Guayaquil, además se roció fipronil al $0.15 \%$ antes de proceder con la necropsia, como norma de bioseguridad para los profesionales que la realizaron.

Una vez capturados los animales se transportaron vivos hasta el laboratorio de Microbiología de la FMVZ de la Universidad de Guayaquil, donde se realizó la necropsia del animal y se extrajo la muestra para posteriormente ser analizado mediante las técnicas reportadas por Aluja y Constantino ${ }^{(17,18)}$.

\section{Análisis de laboratorio}

Las muestras se analizaron por los métodos directos y sedimentación con solución salina saturada $^{(17,19)}$, luego se examinaron al microscopio óptico usando objetivos de 10X y 40X.

Los parámetros a seguir durante la necropsia para la extirpación del aparato gastrointestinal de los roedores fueron los reportados por de Aluja y Constantino ${ }^{(18)}$. Además se realizó la técnica cualitativa de Travassos, con el fin de obtener parásitos $\operatorname{adultos}^{(18,20)}$. Se revisaron las mucosas de los órganos ya lavados para detectar helmintos adultos o juveniles adheridos a la mucosa o por debajo de ella.

\section{Resultados}

Después del armado y cebado de las trampas en los diferentes sitios de estudio, se esperó por un lapso de $12 \mathrm{~h}$, posteriormente al tiempo indicado, se realizó la revisión de las trampas, procediendo a identificar taxonómicamente las especies de roedores ${ }^{(16,17)}$, encontrando un total 87 roedores capturados (40 roedores Rattus rattus y 47 roedores Rattus norvegicus) y analizados, de los cuales 20 (22.99\%) resultaron positivos para $H$. nana y $10(11.49 \%)$ fueron positivos para $H$. diminuta, mediante el método coproparasitario directo y sedimentación con solución salina saturada.

Además, se logró identificar la distribución del parásito en las especies de roedores estudiados; H. nana $12(100 \%)$ y $H$. diminuta (0 \%) en Rathus rathus, mientras que $H$. diminuta $10(55.5 \%)$ y H. nana $8(44.5 \%)$ en Rathus norvegicus.

En todas las áreas estudiadas se encontraron casos de Hymenolepis nana y diminuta. En el área 1, hubo un total de 4 casos de $H$. nana y 2 casos para $H$. diminuta. En el área 2, se presentaron 7 casos de $H$. nana y 3 casos para $H$. diminuta. En el área 3, se determinó un total de 9 casos de $H$. nana y 5 casos para $H$. diminuta (Cuadro 1 ). 
Cuadro 1: Total de los parásitos por área

\begin{tabular}{cccccc}
\hline & Área 1 (\%) & Área 2 (\%) & Área 3 (\%) & Total & $\%$ \\
\hline Hymenolepis nana & $4(4.59)$ & $7(8.04)$ & $9(10.34)$ & 20 & 22.97 \\
\hline Hymenolepis diminuta & $2(2.29)$ & $3(3.44)$ & $5(5.74)$ & 10 & 11.47 \\
\hline
\end{tabular}

Además, se encuestaron 90 personas con preguntas sobre el riesgo de transmisión de enfermedades de roedores a humanos, en donde, todos manifestaron desconocer sobre Hymenolepidiasis; 60 personas $(66.67 \%$ ) manifestaron consumir agua de bidones y 30 (33.33\%) mencionaron comprar agua de tanqueros. Todas las personas encuestadas manifestaron descartar sus excretas por pozo séptico. Para el caso de las excretas de los roedores, los encuestados indicaron suponer que caían al suelo.

Según la presencia de roedores intra y peridomiciliarios, 32 personas $(35.55 \%)$ manifestaron ver roedores intradomiciliarios; 48 personas $(53.33 \%)$ manifestaron ver roedores peridomiciliarios; 10 personas $(11.11 \%)$ manifestaron no ver roedores dentro y fuera de sus casas. Por otro lado, solo 17 personas (18.88\%) declararon tener en sus casas depósitos de alimentos farináceos. Así mismo, 13 personas (14.44 \%) indicaron ver Tenebriónidos en sus depósitos de alimentos farináceos localizados en sus hogares.

Respecto a la situación sanitaria, 70 personas encuestadas $(77.78 \%)$ mencionaron no poseer agua potable y el $100 \%$ de los encuestados manifestaron no contar con alcantarillado.

En relación a los sitios de captura, en el área 1, se determinaron casos de parásitos intestinales en roedores que rondaban áreas como: casas habitadas, solares baldíos, zanjas y basureros. En el área 2, se determinaron parásitos intestinales en roedores que merodeaban en: zanjas y basureros. En el área 3, se determinaron casos de parásitos intestinales en roedores de alrededores de la Unidad Policial Comunitaria (UPC), zanja, basurero y solares baldíos.

Para el cálculo de casas afectadas por la presencia cercana de roedores se utilizó un radio de acción de 40 a 50 m. para la especie Rattus rattus y de 30 a 45 m para Rattus novergicus respectivamente ${ }^{(21)}$.

Dentro del estudio de impacto en la población, se logró evidenciar en el área 1 que los casos de roedores positivos tenían dentro de su perímetro de afectación un promedio de 10 a 18 casas, lo cual sugiere un perjuicio directo de 10 a 18 familias respectivamente. En el área 2. Las trampas donde se encontraron roedores positivos tomando el radio de acción comprometían entre 15 a 18 casas. En el área 3, las trampas donde se encontraron roedores positivos tomando el radio de acción comprometían entre 10 a 25 casas. 


\section{Discusión}

La prevalencia de Hymenolepis nana encontrada del $22.99 \%$, es mayor que la determinada en Costa Rica $(0.97 \%)^{(22)}$; en Cuba $(2.56 \%)^{(23)}$; en Perú $(6.8 \%)^{(17)}$; en Brasil $(8.8 \%)^{(24)}$; en Argentina $(8.2 \%)^{(19)}$. Así mismo, se determinan prevalencias bajas de $H$. nana en países de otros continentes como: en Irán el $2.5 \%{ }^{(25)}$, entre el 3.3 al $10.3 \%$ en China; en Países bajos entre el $3.3 \%$ al $4.1 \%{ }^{(26)}$; pero se ha registrado prevalencias mayores en Taiwán $21.8 \%{ }^{(26)}$, Brasil $35.7 \%(27)$ e Italia $100 \%{ }^{(26)}$. Cabe destacar que la región costera de Ecuador es una zona donde existe una temperatura ambiental alta y constante durante todo el año, debido a la presencia de dos estaciones anuales (inviernoverano), las cuales se caracterizan por el alto grado de humedad y presencia de lluvias moderadas a torrenciales en la primera estación, y escasez de lluvias y altas temperaturas ambientales respectivamente.

El $10.64 \%$ de Himenolepis diminuta determinada es menor que la expresada en Cuba con el $11.5 \%{ }^{(23)}$, en Argentina con el $12.2 \%^{(19)}$, en Perú entre el 25.7 y el $43.8 \%{ }^{(28)}$ y en Costa Rica con el $43.68 \%{ }^{(22)}$. También, se determinó prevalencias bajas de H. diminuta en países de otros continentes como: Taiwán con $6.3 \%{ }^{(26)}$ e Irán con $1.7 \%{ }^{(25)}$, en contraste con prevalencias por encima de la encontrada $(10.64 \%)$ en países como China $(27.8 \%)^{(26)}$, Irán $(38.8 \%)^{(25)}$, Países bajos $(10.2$ a $50 \%)$, Serbia $(30.5 \%)$ e India $(62.5 \%)^{(26)}$.

Es de notable relevancia que en todas las áreas estudiadas se encontraron casos de $H$. diminuta y $H$. nana, parásitos zoonóticos, que en el caso de las personas encuestadas manifestaron desconocer sobre la enfermedad que producen. Por otro lado, el $33.33 \%$ mencionaron comprar agua de tanqueros, todas las personas manifestaron descartar sus excretas por pozo séptico, el $35.56 \%$ manifestaron ver roedores intradomiciliarios y el $53.33 \%$ manifestaron ver roedores peridomiciliarios. Así mismo, el $18.88 \%$ mencionaron tener en sus casas depósitos de alimentos farináceos que son principal fuente de Tenebriónidos, que son hospederos intermediarios de la $H$. diminuta ${ }^{(29)}$, lo que podría conllevar a la aparición de casos en humanos, sobre todo en la época invernal cuando el número de vectores aumenta ${ }^{(25)}$.

El $77.78 \%$ de los encuestados mencionaron no poseer agua potable, y también todos los encuestados manifestaron no contar con alcantarillado, lo cual es un riesgo potencial para el incremento de roedores frente al presente incremento de la población ${ }^{(21)}$, a su vez, estos últimos mencionados son indicadores importantes de parasitosis desatendidas en poblaciones vulnerables ${ }^{(7,30)}$.

Finalmente, se determinaron casos de parásitos intestinales en roedores de casas habitadas, zanjas, basureros, solares y terrenos baldíos que son sitios en donde hay mayor proliferación de estos mamíferos ${ }^{(21)}$. 


\section{Conclusiones e implicaciones}

Este estudio determinó la presencia de $H$. diminuta y $H$. nana en heces de roedores de la ciudadela "Las Piñas" del cantón Milagro, así mismo, se concluye que es evidente la presencia de estos parásitos helmintos en el sitio de estudio, lo que constituye un problema de salud pública y existe el riesgo de presentarse nuevos casos. Por otra parte, se hace hincapié en fomentar un estudio epidemiológico a fondo para identificar los miembros más vulnerables dentro de los núcleos familiares, para el desarrollo de campañas de concientización dirigidas a grupos etarios con riesgo de sufrir la parasitosis.

\section{Agradecimientos y conflicto de interés}

Se agradece la colaboración de la Facultad de Medicina Veterinaria y Zootecnia de la Universidad de Guayaquil y a su vez al Comité Pro mejoras Nuevos Horizontes de "Las Piñas", cuya participación y apertura al presente estudio permitió develar los datos expuestos.

\section{Literatura citada:}

1. Han BA, Schmidt JP, Bowden SE, Drake JM. Rodent reservoirs of future zoonotic diseases. Proc Nat Academy Sci. United States of America. 2015;112(22):70397044.

2. Karesh WB, Dobson A, Lloyd-Smith JO, Lubroth J, Dixon MA, Bennett M, et al. Ecology of zoonoses: natural and unnatural histories. Lancet (London, England). 2012;380(9857):1936-1945.

3. Franssen F, Swart A, van Knapen F, van der Giessen J. Helminth parasites in black rats (Rattus rattus) and brown rats (Rattus norvegicus) from different environments in the Netherlands. Infect Ecol Epidemiol 2016;6:1-14.

4. Reperant LA, Hegglin D, Tanner I, Fischer C, Deplazes P. Rodents as shared indicators for zoonotic parasites of carnivores in urban environments. Parasitology 2009;136(3):329-337.

5. Acuña A, Calegari L, Cuto S, Lindner C, Rosa R, Salvatella R. Helmintiasis intestinal. Manejo de las Geohelmintiasis http://www.higiene.edu.uy/parasito/parasitologia_archivos/GEOHELMI\%20para\% 20medicos.pdf 2003; 1(1):[42].

6. Kenyon PA, Roses PR. Regional approaches to neglected tropical diseases control in Latin America and the Caribbean. Institute of Medicine Forum on Microbial Threats. Washington, DC. USA: National Academies Press; 2011.

7. WHO. Investing to overcome the global impact of neglected tropical diseases. EEUU: STAG-NTD; 2015. 
8. Zonta ML, Navone GT, Oyhenart EE. Parasitosis intestinales en niños de edad preescolar y escolar: situación actual en poblaciones urbanas, periurbanas y rurales en Brandsen, Buenos Aires, Argentina: Parasitol Latinoam 2007;62:54-60.

9. Llop A, Valdés M, Zuazo J. Microbiología y parasitología médicas. 1a Ed, La Habana-Cuba: Editorial de Ciencias Médicas; 2001.

10. Hernández W. Determinación de la presencia de Hymenolepis nana e Hymenolepis diminuta en roedores sinantrópicos, en la comunidad Los Lirios, Masagua, Escuintla, Guatemala [Tesis]. Guatemala: San Carlos de Guatemala; 2016.

11. Mikhail MW, Metwally AM, Allam KA, Mohamed AS. Rodents as reservoir host of intestinal helminthes in different Egyptian agroecosystems. J Egyptian Soc Parasitol 2009;39(2):633-640.

12. Martínez I, Gutiérrez M, Aguilar J, Shea M, Gutiérrez M, Ruíz L. Infección por Hymenolepis diminuta en una estudiante universitaria. Rev Biomed 2012;23(1):6164.

13. Rossomando MJ, Márquez W, Prado J, Chacón N. Epidemiología de himenolepiosis y otras parasitosis intestinales en una comunidad suburbana de Escuque, TrujilloVenezuela. Rev Fac Med 2008;31:101-110.

14. Municipio-Milagro. Características ecológicas del cantón Milagro y descripción de la ciudadela "Las Piñas". Pazmiño B, editor. Municipio del cantón Milagro. 2018.

15. Milagro-Map. Mapa satelital del cántón Milagro. 2018.

16. Hancke D, Navone GT, Suarez OV. Endoparasite community of Rattus norvegicus captured in a shantytown of Buenos Aires City, Argentina. Helminthologia 2011;48(3):167.

17. De Sotomayor R, Serrano E, Tantaleán M, Quispe M, Casas G. Identificación de parásitos gastrointestinales en ratas de Lima Metropolitana. Rev Inv Vet Perú 2015;26(2):273-281.

18. Aluja A, Constantino F. Técnicas de necropsia en animales domésticos. 2da. ed. Mexico: Editoral el Manual Moderno; 2002.

19. Fitte B, Robles MR, Dellarupe A, Unzaga JM, Navone GT. Hymenolepis diminuta and Rodentolepis nana (Hymenolepididae: Cyclophyllidea) in urban rodents of Gran La Plata: association with socio-environmental conditions. J Helminthol 2018;92(5):549-553.

20. Leguia G, Casas E. Enfermedades parasitarias y atlas parasitológico de camélidos sudamericanos. Lima: Editorial de Mar; 1999.

21. OPS. Manual para el control integral de roedores. Colombia: Ministerio de Salud y Protección Social y OPS; 2012. 
22. Vives N, Zeledón R. Observaciones parasitológicas en ratas de San José, Costa Rica. Rev Biol Trop 1957;5(2):173-194.

23. Companioni-Ibañez A, Atencio-Millán I, Cantillo-Padrón J, Hernández-Contreras N, González-Rizo A, Núñez-Fernández F. Prevalencia de endoparásitos en roedores sinantrópicos (Rodentia: Muridae) en una localidad de La Habana, Cuba. Rev Cubana Med Trop 2016;68:240-247.

24. Simoes RO, Luque JL, Gentile R, Rosa MC, Costa-Neto S, Maldonado A. Biotic and abiotic effects on the intestinal helminth community of the brown rat Rattus norvegicus from Rio de Janeiro, Brazil. J Helminthol 2016;90(1):21-27.

25. Arzamani K, Salehi M, Mobedi I, Adinezade A, Hasanpour H, Alavinia M, et al. Intestinal Helminths in Different Species of Rodents in North Khorasan Province, Northeast of Iran. Iranian J Parasitol 2017;12(2):267-273.

26. Yang D, Zhao W, Zhang Y, Liu A. Prevalence of Hymenolepis nana and H. diminuta from Brown Rats (Rattus norvegicus) in Heilongjiang Province, China. Korean J Parasitol 2017;55(3):351-355.

27. Chagas CRF, Gonzalez IHL, Favoretto SM, Ramos PL. Parasitological surveillance in a rat (Rattus norvegicus) colony in Sao Paulo Zoo animal house. Ann Parasitol 2017;63(4):291-297.

28. Abad D, Chávez A, Pinedo R, Tantaleán M, González O. Helmintofauna gastrointestinal de importancia zoonótica y sus aspectos patológicos en roedores (Rattus spp) en tres medioambientes. Rev Inv Vet Perú 2016;27(4):376-750.

29. Makki MS, Mowlavi G, Shahbazi F, Abai MR, Najafi F, Hosseini-Farash BR, et al. Identification of Hymenolepis diminuta Cysticercoid Larvae in Tribolium castaneum (Coleoptera: Tenebrionidae) beetles from Iran. J Arthropod-borne Diseases 2017;11(2):338-43.

30. World Health Organization. (WHO). Neglected tropical diseases: hidden successes, $\begin{array}{lll}\text { emerging } & \text { opportunities. } & \\ & \text { USA } & \end{array}$ http://www.who.int/neglected_diseases/resources/9789241598705/en/. 